\title{
A preliminary approach to study the behavior of human fingertip at contact via experimental test and numerical model
}

\author{
Maria Laura D'Angelo ${ }^{1}$, Ferdinando Cannella ${ }^{1}$, Mariapaola D'Imperio ${ }^{1}$, Matteo Bianchi ${ }^{1,2}$ \\ ${ }^{1}$ Department of Advanced Robotics, Istituto Italiano di Tecnologia, Via Morego 30, 16163 Genova, Italy \\ ${ }^{2}$ Research Center "E.Piaggio", School of Engineering, University of Pisa, 56126 Pisa, Italy
}

\begin{abstract}
How human fingertip deforms during the interaction with the environment represents a fundamental action that shapes our perception of external world. In this work, we present the proof of concept of an experimental in vivo set up that enables to characterize the mechanical behavior of human fingertip, in terms of contact area, force and a preliminary estimation of pressure contour, while it is put in contact against a flat rigid surface. Experimental outcomes are then compared with the output of a 3D Finite Element Model (FEM) of the human fingerpad, built upon existing validated models. The good agreement between numerical and experimental data suggests the correctness of our procedure for measurement acquisitions and finger modeling. Furthermore, we will also discuss how our experimental data can be profitably used to estimate strain limiting deformation models for tactile rendering, while the here reported 3D FE model has also been profitably employed to investigate hypotheses on human tactile perception.
\end{abstract}

Section: RESEARCH PAPER

Keywords: biomechanics; pressure sensors; pressure distribution; contact area; finite element model; fingertip at contact

Citation: Maria Laura D'Angelo, Ferdinando Cannella, Mariapaola D'Imperio, Matteo Bianchi, A preliminary approach to study the behavior of human fingertip at contact via experimental test and numerical model, Acta IMEKO, vol. 6, no. 2, article 15, July 2017, identifier: IMEKO-ACTA-06 (2017)-02-15

Section Editor: Paul Regtien, Measurement Science Consultancy, The Netherlands

Received June 5, 2016; In final form April 29, 2017; Published July 2017

Copyright: @ 2017 IMEKO. This is an open-access article distributed under the terms of the Creative Commons Attribution 3.0 License, which permits unrestricted use, distribution, and reproduction in any medium, provided the original author and source are credited

Funding: European Community funded project WEARHAP (no. 601165) and by the ERC Advanced Grant no. 221166 SoftHands

Corresponding author: Maria Laura D’Angelo, e-mail: marialaura.dangelo@iit.it

\section{INTRODUCTION}

The investigation of the mechanisms determining human tactile perception represents a fundamental topic in haptics (i.e. the science and the technology of touch) community. Indeed, if the comprehension of the underpinning human perceptual mechanisms is clearly the main goal of many neuroscientific studies, it could also lead to a correct development of tactile devices and haptic systems, as they are intended to convey controllable and effective stimuli.

What is noticeable is that the mandatory step to properly develop such investigation is the clear understanding of mechanical properties of soft tissues and, more specifically, the tissues of the main "organ of Touch", i.e., human hand and its fingers. Indeed, tactile stimuli are mainly mechanical as the inputs eliciting the response of tactile receptors [1]. In literature, we can find different approaches for in vivo acquisition of mechanical measurements related to human fingerpad (see e.g.
[2], [3] and [4]) via different systems, such as digital camerabased techniques to measure skin deformation [5], Magnetic Resonance (MR) images taken before and during compressional loading of finger tissues [6] or non-intrusive suction instruments [7], among the others.

Previous studies have determined the mechanical behaviour of human fingerpad under quasi-static and cycling loading, using indenters of various shapes and sizes [8]. Furthermore, at neurophysiological level, other works focused on the relationship between fingerpad mechanoreceptors response and different static and dynamic mechanical load stimuli [1], [4] and [9]. Looking at non-invasive methods for fingerpad pressure measurements (at the contact between the fingertip and the indenters) [10], they leverage upon different techniques, e.g. pressure sensitive films to determine the peak of force and the pressure centroid during the grasping of objects [11], or flat 
capacitive array sensors to determine the whole pressure distribution on the human fingerpad [12].

However, to the best of our knowledge none of these aforementioned studies have characterized fingertip behaviour at contact through experimental measurement including contact area, force and pressure distribution, simultaneously, as we report in this work.

These in vivo measurements are then commonly used to develop Finite Element Models (FEM) of the human finger, which represent useful tools adopted by the haptic research community to investigate some properties that are hardly measurable. In practice, a FEM of human fingerpad indentation response could be verified against the in vivo biomechanical data. Many numerical models have been proposed in literature, which differ for dimensionality, accuracy and mechanical response tailored on different tasks (see e.g. [3], [4], [14], [15] and [16] among the most significant). However, despite individual differences, all these FEMs need to be first validated, or, in other words, to be tested in terms of the matching between numerical and experimental results for a large variety of mechanical conditions and parameters. For these reasons, an exhaustive investigation of the mechanical characteristic response of the fingerpad and the acquisition of in vivo data is usually strongly required.

In particular, the usage of matrices-array that are able to map force and position over a continuous area or thin and flexible single zone Force Sensing Resistor (FRS), are really common for those situations where we have high pressure and wide contact area as biomedical applications. For example, palm, plantar and car seat pressure distribution measurements [17], [18] and [19] (e.g. by Novel (St. Paul, MN), Tekscan (Boston, MA) and Sensitronics (Bow, WA)) are used to investigate grasping, walking and seating forces. The effectiveness of these sensors relies on their low stiffness and high sensitivity.

In this paper, we propose an innovative experimental test-rig that can measure in vivo finger deformation, contact and pressure area, while a flat rigid plate is pushed against the fingertip in a controllable fashion. Contact area is obtained through vision-based techniques, while pressure contact area information is acquired through a pressure film sensor in paperboard material with $0.05 \mathrm{mN} \cdot \mathrm{m}$ stiffness and $10 \mathrm{kPa}$ of threshold [22]. This pressure sensor, made by a film of microscopic pigmented particles adhered to the donor substrate, can adapt to the high deformation of the fingertip (whose compliance is around $0.1 \mathrm{mN} \cdot \mathrm{m}$ ) [20], [21] that undergoes during the contact of the fingerpad with the external flat rigid surface. In this way, the microscopic particles were attracted to the chemically surface-treated receiver sheet, only when the pressure sensor threshold is exceeded (higher than 10 $\mathrm{kPa})$.

Our aim is to present a proof of concept of an integrated acquisition system, which allows to study the fingerpad mechanical properties under a specific condition. These experimental measures are then compared with the numerical outcomes of a FE model of human fingertip that we built upon existing validated models and data in literature (Gerling et al. [15] and Wu et al. [16]).

The experimental data we collected have been also applied to correctly compute deformations and contact forces for haptic rendering [23]. Furthermore, the model we propose in this work, which exhibits a good agreement with our experimental data, has been used for the investigation of human touch perceptual mechanisms [24].
This paper is divided in two parts: the first one describes the experimental set-up of the test-rig for the measurement of contact area, pressure area (i.e. the area with a pressure above the sensor threshold that is equal to $10 \mathrm{kPa}$ ) and contact forces (using a 6 DoF force/torque sensor) during experiments of finger indentation. The contact area can be obtained with a high-resolution camera placed beneath the flat rigid plate, using a luminosity threshold-based approach. On the contrary, pressure area is defined as the footprint area over the sensing film. In the second part, we discuss the comparison of our experimental measurements and numerical outputs provided by our FE model of human fingertip built upon literature.

Finally, we show how the here reported experimental data can be profitably employed to numerically investigate some perceptual hypotheses of human touch perception and how the 3D FE model can be also used for the estimation of parameters for haptic rendering applications.

\section{MATERIALS AND METHODS}

During the experimental acquisitions, we indented the subject's fingertip with a flat surface using a linear actuator, and we measured fingertip area size, deformation and pressure distribution. Contact area measurements were carried out in two different ways: i) the contact area was obtained through high resolution video-camera (see details in the next section), ii) the area of the pressure distribution was obtained from the pressure fingertip print on the pressure sensing film. Both types of measurements were used for the comparison with a $3 \mathrm{D}$ Finite Element (FE) model of human fingerpad. To perform the experiments, we built the experimental test rig shown in Figure 1 and described in the next section.

\subsection{Test-rig Instrumentation}

The test-rig is a one degree of freedom (DoF) device that permits to move a flat rigid surface towards the fingertip and to deform/indent it in a controllable fashion. The displacement is given by a linear DC actuator (Faulhaber LM2070-040-01 plus MCLM3003 controller) with $9.2 \mathrm{~N}$ of constant force, max acceleration of $94 \mathrm{~m} / \mathrm{s}^{2}, 200 \mu \mathrm{m}$ of accuracy and $60 \mu \mathrm{m}$ of repeatability. The contact area is recorded by a video-camera (Sony HDRCX505VE 12MP) with 1500 x 1120 effective pixels per video frame and 50 fps. The one direction movement is guaranteed by the slider (Standa 7T125Z-10), which has an angular deviation $<200 \mu \mathrm{rad}$. The perpendicular force $[\mathrm{N}$,

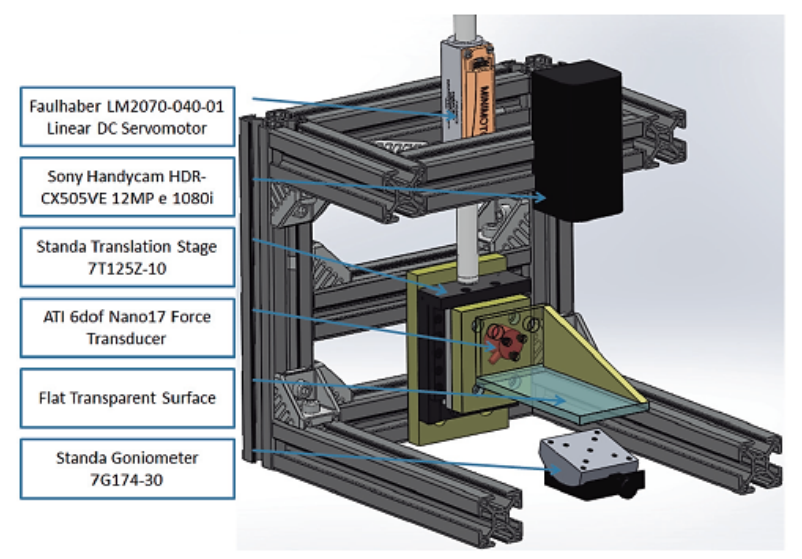

Figure 1. Test-rig instrumentation system. 
acquired during each experimental test, is recorded using the 6 DoF force/torque sensor (ATI 6 DoF Nano17) with $0.00625 \mathrm{~N}$ of resolution for force and $0.03125 \mathrm{Nmm}$ for torque. Using this $6 \mathrm{DoF}$ sensor we were sure to take under control also the undesired force components that were not perpendicular. The force sensor is rigidly connected to the flat rigid surface (indenter), which is moved against the fingerpad. Pressure is measured with a film pressure Pressurex-micro Green (Sensor Products Inc. PMG1), with $10-400 \mathrm{kPa}$ pressure range and 45 $\mu \mathrm{m}$ spatial resolution. The Pressurex-micro Green sensor is extremely thin and flexible $(0.2 \mathrm{~mm})$, thus conforming to curved surfaces [22]. It is simple to use and it is ideal for noninvasive applications. The procedure that we used during the experiments was:

i) the sensor was correctly positioned between the human fingerpad and the flat rigid surface;

ii) the flat surface was moved in a controllable fashion against the fingerpad;

iii) the film pressure sensor was removed and the pressure distribution profile, which arose from the contact between the two media, revealed.

Moreover, since the human fingerpad measurements in terms of force $[\mathrm{N}]$, contact and pressure area $\left[\mathrm{mm}^{2}\right]$ are very influenced by the fingertip orientation into the fingerholder, we employed a micro goniometer (Standa 7G174-30) with reading resolution of $0.1^{\circ}$ in the measurement setup to guarantee uniform and repeatable experimental conditions. The measurement scheme used during the experimental tests is depicted in Figure 2 and Figure 3.

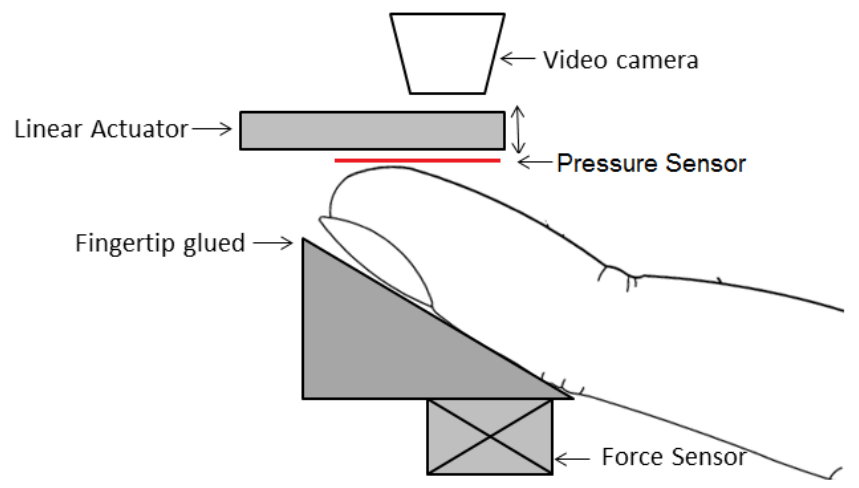

Figure 2. Measurement scheme during experimental tests.

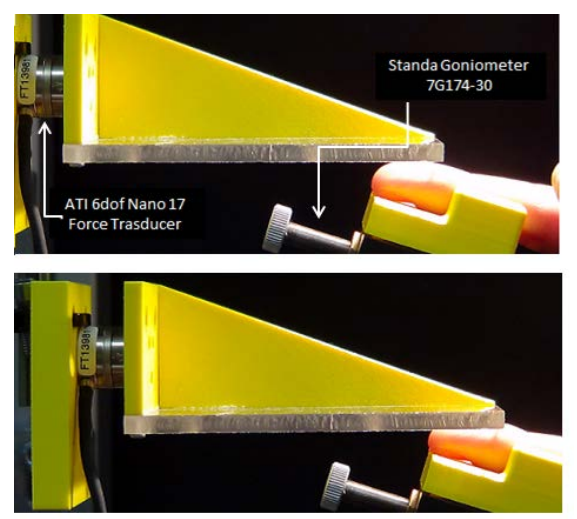

Figure 3. Positioning of the fingertip in the finger-holder; the flat surface was moving against the fingerpad.

\section{EXPERIMENTAL TEST PROCEDURE}

During each experimental test, we positioned the fingertip in the finger-holder and moved the flat Plexiglas surface toward the fingerpad, as shown in Figure 3. To prevent losing contact during the experiment, the finger was placed within a custommade finger-holder and fixed to it on the top of the nail and was oriented at $15^{\circ}$ w.r.t. the flat surface. The fingerpad was free of callus and subject (right-handed female, age 28) gave her informed consent to the experimental test.

We performed three experimental tests indenting the fingertip at three different levels of indentation $(1 \mathrm{~mm}, 2 \mathrm{~mm}$ and $3 \mathrm{~mm}$ ), while the force sensor and the video-camera recorded the force values and contact area, respectively. The linear actuator can control both the indentation velocity and displacement of the flat indenter. Three different displacement levels were considered ( $1 \mathrm{~mm}, 2 \mathrm{~mm}$ and $3 \mathrm{~mm}$ ) and only one single velocity $(2 \mathrm{~mm} / \mathrm{s})$, as a preliminary test case. We tested the participant's fingertip under the different experimental conditions the same day, in the morning, when the fingertip skin was not affected by any mechanical stress or temperature variation due to the daily duties. Some results of the images captured by video-camera are displayed in Figure 4, where four snapshots show the different contact areas per each indentation displacement $(0,1 \mathrm{~mm}, 2 \mathrm{~mm}$ and $3 \mathrm{~mm}$, respectively). Once the high-resolution video-camera images were acquired, the fingertip contact area was measured based on an automated algorithm, built in Matlab, which will be discussed in the next section.

\subsection{Contact Area Values and Force}

In order to not saturate the fingertip mechanoreceptors response, the finger displacement was along a vertically downward direction with a displacement from 0 to $3 \mathrm{~mm}$ [8]. Moreover, since we wanted to estimate only the elastic properties of the fingerpad skin, minimizing the effect of viscosity, we selected a very low velocity for the indenter (2 $\mathrm{mm} / \mathrm{s}$ ). The contact area was recorded, when the fingertip skin was completely stuck to the Plexiglas surface. Since the dimension of the subject's fingerpad who carried out the experimental tests was $15.5 \mathrm{~mm}$ x $12.8 \mathrm{~mm}$, we performed a calibration setup positioning the high-resolution video-camera at $100 \mathrm{~mm}$ of distance from the human fingerpad and we chose a video-camera resolution equal to $1500 \times 1120$ pixels. In this way, we obtained that 1 pixel corresponds to a square with a dimension of $0.0393 \mathrm{~mm}\left(0.0015 \mathrm{~mm}^{2}\right)$. Using the
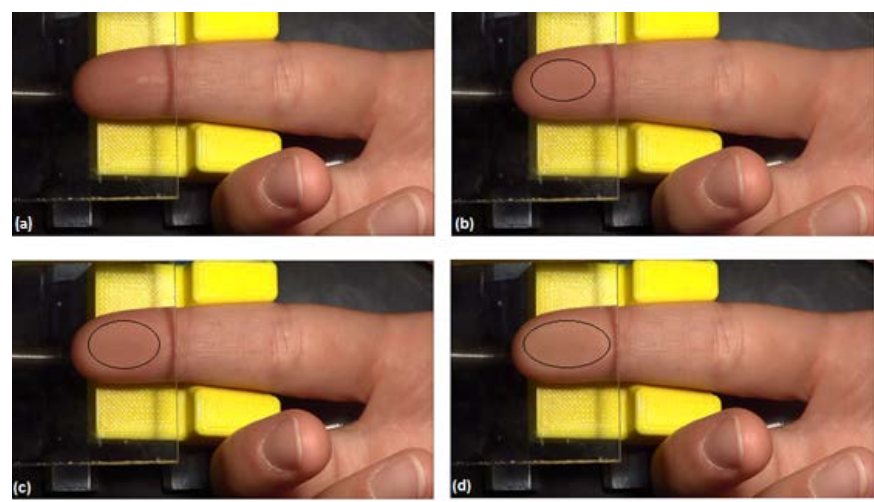

Figure 4. Contact area at different indentation values: a) $0 \mathrm{~mm}$, b) $1 \mathrm{~mm}, \mathrm{c}$ ) $2 \mathrm{~mm}$ d) $3 \mathrm{~mm}$. 
aforementioned pixel dimension, the imaging post-processing was performed on images with $400 \times 350$ pixels. Once we collected all fingertip indentation images, the post-processing consists of three main steps:

1) for each RGB image, we generated a grey scale image and then we visualized the image pixel distribution, through a pixel intensity histogram.

2) in order to focus only on the subject's fingertip contact area, we chose a threshold value: we assigned to the pixels whose grey intensity value was above the threshold (value = 50), the intensity level of 0 (black) and hence they were discarded. In this way only the fingertip contact area pixels were considered for the image post-processing phase [28].

3) The value of the contact area, between the fingertip and the flat window, can be obtained by summing all the pixels whose intensity was different from 0 (black). As previously mentioned, based on the camera and lens characteristics, one pixel has an area of $0.0015 \mathrm{~mm}^{2}$.

To determine the threshold (discussed at point 2), we performed preliminary measurements, where we used a five cent coin as ground truth and placed it over the flat rigid surface; while the high-resolution camera acquired the images, the area of contact was estimated using our algorithm in Matlab. We found that a threshold of 50 for the intensity ensured a good agreement between the estimated contact area and the geometric one (error less than $2 \%$ ).

In this manner, we obtained all contact area values, which are reported in Table 1. Mean areas and standard deviation, between the three experimental tests (per each indentation value), are also reported in Table 1 . The mean values are obtained as the average of the results across the three tests we performed for each displacement interval. The force values recorded during these tests are reported in Table 2, together with their peak force values.

\subsection{Pressure Distribution Area Values}

The pressure sensor was made by a film of microscopic pigmented particles adhered to the donor substrate, which are

Table 1. Contact areas value $\left[\mathrm{mm}^{2}\right]$ of the indentation with related average and standard deviation (STD).

\begin{tabular}{|c|c|c|c|}
\hline \multicolumn{4}{|c|}{ Experimental Area Values $\left[\mathrm{mm}^{2}\right]$} \\
\hline Indentation & $1 \mathrm{~mm}$ & $2 \mathrm{~mm}$ & $3 \mathrm{~mm}$ \\
\hline Test 1 & 92.82 & 148.8 & 147.2 \\
\hline Test 2 & 70.07 & 117.8 & 129.6 \\
\hline Test 3 & 99.19 & 106.6 & 121.6 \\
\hline Average & 87.36 & 124.4 & 132.8 \\
\hline STD & 15.31 & 21.86 & 13.10 \\
\hline
\end{tabular}

Table 2. Contact force values [N] of the indentation with related average and standard deviation (STD).

\begin{tabular}{|c|c|c|c|}
\hline \multicolumn{4}{|c|}{ Experimental Force Values [N] } \\
\hline Indentation & $1 \mathrm{~mm}$ & $2 \mathrm{~mm}$ & $3 \mathrm{~mm}$ \\
\hline Test 1 & 0.80 & 1.01 & 1.72 \\
\hline Test 2 & 0.75 & 1.05 & 1.76 \\
\hline Test 3 & 0.78 & 1.02 & 1.64 \\
\hline Average & 0.78 & 1.03 & 1.71 \\
\hline STD & 0.03 & 0.02 & 0.06 \\
\hline
\end{tabular}

attracted to the chemically surface-treated receiver sheet. The chemical compatibility between the receiver sheet and the donor sheet causes intermolecular diffusion that creates an interpenetrating network between the pigment donor and the receiver coating. Upon separation, micro-shear forces compel the particles to dislodge from the pigmented donor substrate, leaving an impression [22].

The film pressure sensor was inserted between the fingerpad and the flat moving surface (see Figure 2) and changed for each measurement. The pressure sensor full scale is sufficient for our application. However, considering the film pressure sensor threshold $(10 \mathrm{kPa})$ and resolution $(15 \mathrm{kPa})$, we cannot expect an output with more than two levels of pressure (lower and greater than $10 \mathrm{kPa}$ ), since the maximum expected value is around 25 $30 \mathrm{kPa}[25]$.

Note that our goal is to provide a proof of concept of the experimental test-rig, which enables to acquire, simultaneously and for the first time, displacement, force, contact and pressure area. The area with a pressure above the threshold is named "pressure area". Results of pressure area, obtained during the experimental tests from the pressure sensor film, for each displacement interval, are shown in Figure 5. From left to right $(1 \mathrm{~mm}$ to $3 \mathrm{~mm})$, it is possible to observe the pigmented particle (red particles) that adhered to the donor substrate, during the fingertip indentation against the flat Plexiglas surface.

Since the pressure sensor threshold is $10 \mathrm{kPa}$ and the resolution is $15 \mathrm{kPa}$, we have highlighted only the pressure area at $2 \mathrm{~mm}$ and $3 \mathrm{~mm}$ of indentation level, with a dashed white line; while the pressure value at $1 \mathrm{~mm}$ of indentation was less than $10 \mathrm{kPa}$.

Pressure area values, obtained from the pressure sensor film, were manually measured. At the end of each experimental test, the post-processing procedure was the following one:

1) each film pressure sensor was scanned using a normal scanner and the image post-processing was performed in Matlab;

2) per each fingertip pressure area, the length and the width at each indentation level (1 $\mathrm{mm}, 2 \mathrm{~mm}$ and $3 \mathrm{~mm})$ was measured using a ruler;

3) finally the pressure area was obtained and calculated considering the fingerprint on the film pressure sensor as an ideal ellipse.

Pressure area results, with average and standard deviation, are summarized and shown in Table 3.

Some preliminary results, related to both contact area and pressure area, obtained from the post-processing phase, are

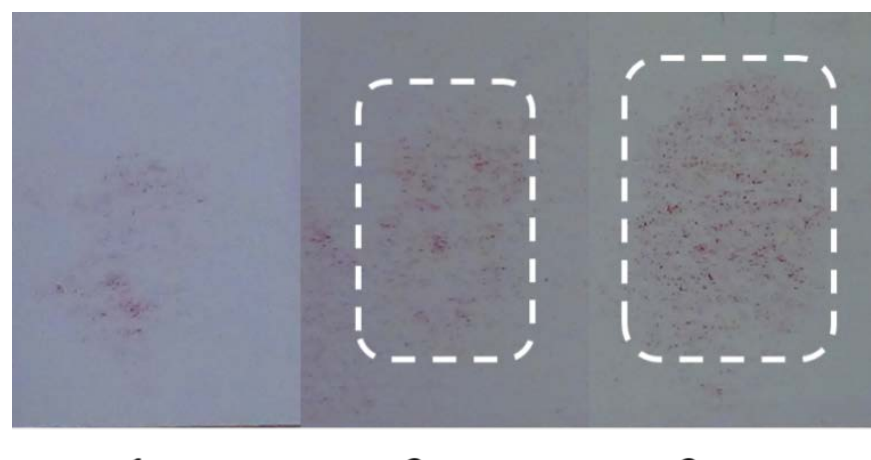

$1 \mathrm{~mm}$

$2 \mathrm{~mm}$

$3 \mathrm{~mm}$

Figure 5. Pressure contact area with different indentation: $1 \mathrm{~mm}, 2 \mathrm{~mm}$ and $3 \mathrm{~mm}$, obtained from the pressure sensor film. 
Table 3. Pressure area values of the indentation with related average and standard deviation.

\begin{tabular}{|c|c|c|c|}
\hline \multicolumn{4}{|c|}{ Experimental Pressure Area $\left[\mathrm{mm}^{2}\right]$} \\
\hline Indentation & $1 \mathrm{~mm}$ & $2 \mathrm{~mm}$ & $3 \mathrm{~mm}$ \\
\hline Test 1 & 40.3 & 87.8 & 104.1 \\
\hline Test 2 & 37.8 & 77.0 & 82.0 \\
\hline Test 3 & 49.0 & 61.6 & 79.7 \\
\hline Average & 42.3 & 75.4 & 88.6 \\
\hline STD & 5.88 & 13.1 & 13.4 \\
\hline
\end{tabular}

shown in Figure 6. From top to bottom, it is possible to notice the subject's finger and the contact area images, the latter achieved via the high-resolution camera. line).

It is also possible to observe the pressure area (yellow dotted

\section{FINITE ELEMENT (FE) MODEL}

The numerical model is a fully parametrized Finite Element (FE) model that replicates the fingertip shape of right-handed subject (female, age 28), who carried out experimental tests.

\subsection{Geometry and Materials}

The geometry of the fingertip was determined by the physical one of the fingertip of the subject. We built a model in ANSYS [26], in order to simulate the shape and structure of a human distal phalanx. The fingertip was assumed to be composed of two skin layers (epidermis and dermis), subcutaneous tissue, bone and nail as shown in Figure 7.

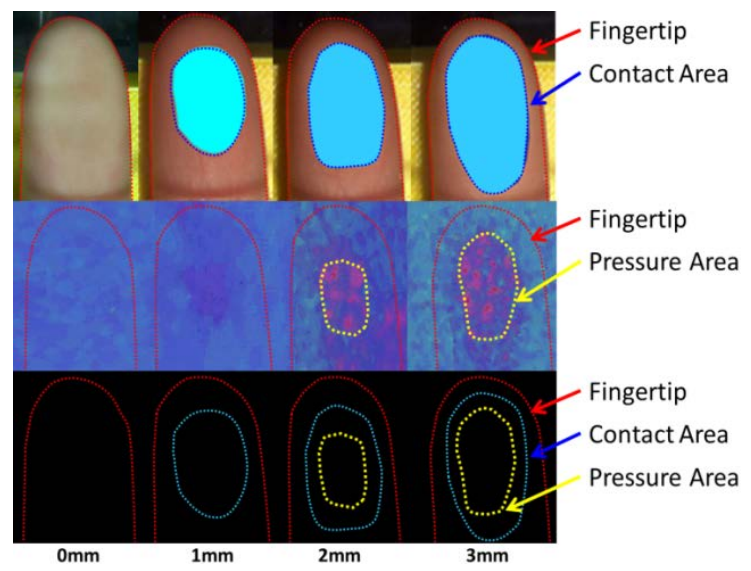

Figure 6. (top) Contact area (dotted cyan line) measured through high resolution camera. (middle) Pressure area (dotted yellow line) measured with film pressure sensor. (bottom) Comparison between contact and pressure area. From left to right: $0 \mathrm{~mm}$ (no contact), $1 \mathrm{~mm}, 2 \mathrm{~mm}, 3 \mathrm{~mm}$ indentation.

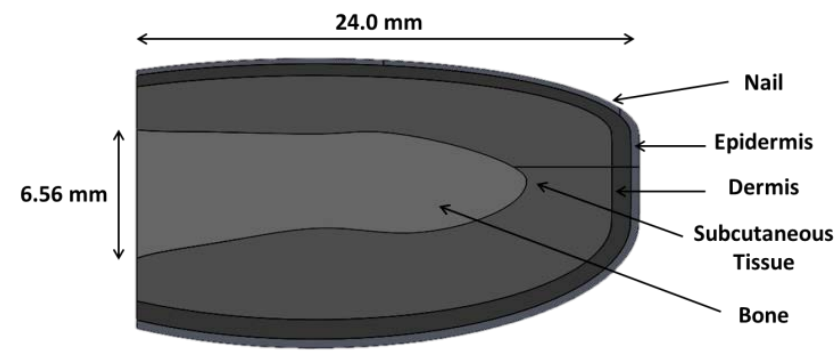

Figure 7. Finger 3D CAD geometry.
The fingertip materials used in the model were the same of Gerling et al. [15] and Wu et al. [16]. In particular, we modelled epidermis, bone and nail as linear materials, while dermis and subcutaneous tissue were modelled as Mooney-Rivlin hyperelastic material [15]. The mechanical properties of these materials are reported in Table 4 and Table 5, respectively. Moreover, the viscoelastic behaviour of the subcutaneous tissue is described in (1), where $W$ represents the mechanical strain energy, $C_{i j}$ are invariant related to the distortional response while $D$ is a constant linked to the volumetric response, $I_{1}$ and $I_{2}$ respectively are the first two deformation invariants.

$W=\Sigma_{i} \Sigma_{j} C_{i j}\left(I_{1}-3\right)^{3}\left(I_{2}-3\right)^{j}+D(J-1)^{2}$.

The FE model of fingertip has 7736 solid brick 8 node elements, with 21714 DoF as shown in Figure 8.

The numerical fingertip model was indented using an inclined plane of $15^{\circ}$ w.r.t the finger longitudinal axis. The parameters of the flat rigid surface, in terms of Young Modulus and Poisson Ratio were $71.3 \mathrm{GPa}$ and 0.17 , respectively. The displacement of the plane is perpendicular to the plane axis, as in the test-rig. Fixed constrain was set on the nail. The contact was established between the moving plate and the bottom part of the finger.

\subsection{Numerical Simulations}

The simulations were carried out moving the flat surface against the fingertip as in the experimental tests, adopting a quasi-static analysis. In Figure 9, some images of the simulations are reported. From left to right, it is possible to note an increasing of the indentation and the contact area. The contours display the pressure level between the plane and the skin. The fingertip pressure areas are manually measured from the numerical output obtained by ANSYS software [26], as shown in Figure 9. In other words, per each level of indentation: $(1 \mathrm{~mm}, 2 \mathrm{~mm}$ and $3 \mathrm{~mm}$ ) we manually measured

Table 4. Mechanical properties of the linear elastic materials.

\begin{tabular}{|c|c|c|}
\hline Material & Young Modulus [MPa] & Poisson Ratio \\
\hline Epidermis & 2.0 & 0.30 \\
\hline Bone & 17.0 & 0.30 \\
\hline Nail & 170.0 & 0.30 \\
\hline Plate & 71300 & 0.17 \\
\hline
\end{tabular}

Table 5. Mechanical properties for the Mooney-Rivlin model materials.

\begin{tabular}{|c|c|c|c|c|c|c|}
\hline Material & $\mathrm{C}_{10}$ & $\mathrm{C}_{01}$ & $\mathrm{C}_{20}$ & $\mathrm{C}_{11}$ & $\mathrm{C}_{02}$ & $\mathrm{D}_{1}$ \\
\hline Dermis & 2430 & 5420 & 239000 & 262000 & 74700 & 13.3 \\
\hline $\begin{array}{c}\text { Subcutaneous } \\
\text { Tissue }\end{array}$ & 300 & 671 & 29800 & 32700 & 9330 & 106.5 \\
\hline
\end{tabular}

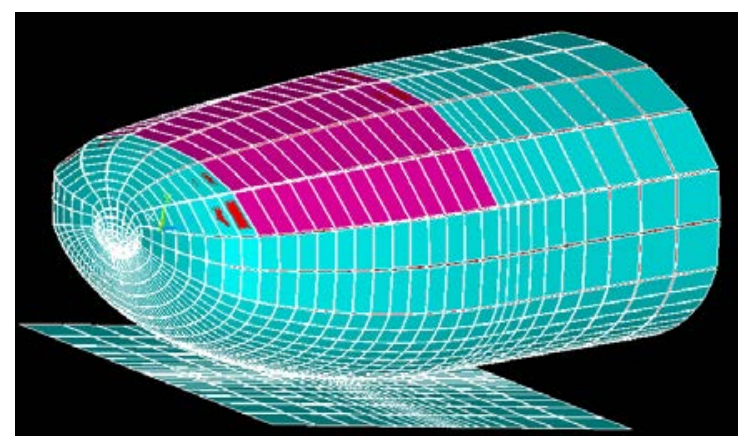

Figure 8. Human fingerpad Finite Element (FE) Model. 


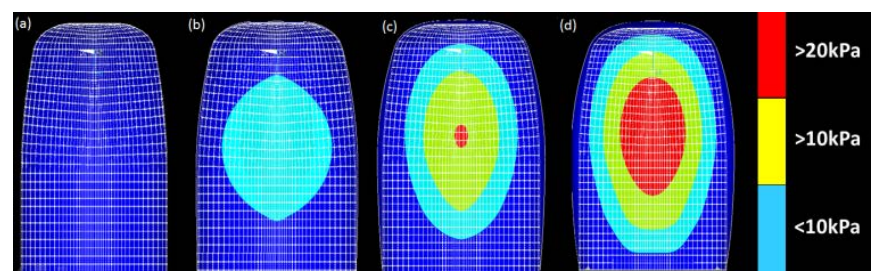

Figure 9. Numerical pressure distribution results with different indentation: a) $0 \mathrm{~mm}$, b) $1 \mathrm{~mm}$, c) $2 \mathrm{~mm}$, d) $3 \mathrm{~mm}$. The cyan area is comparable to the contact area of the experimental results as well as the yellow area to the pressure area.

the length and the width of each pressure area (e.g. $<10 \mathrm{kPa},>$ $10 \mathrm{kPa}$ and $>20 \mathrm{kPa}$ ) and then, as for the contact area, it was approximated to an ellipse.

Finally, the numerical pressure area, obtained from the numerical simulations were compared to the ones obtained from the pressure sensor film, already reported in Table 3.

\section{RESULTS}

The results, in terms of force, contact and pressure area, obtained from experimental tests, are then compared with the numerical outputs of a 3D FE model of the finger, which we built in ANSYS [26].

Our FE fingertip model was used to find information on pressure distribution within fingerpad tissues that cannot be achieved experimentally. The FE numerical outputs of force $[\mathrm{N}]$, contact and pressure area $\left[\mathrm{mm}^{2}\right]$ were reported and then compared with the experimental ones, as shown in Table 6 .

Per each level of indentation ( $1 \mathrm{~mm}, 2 \mathrm{~mm}$ and $3 \mathrm{~mm}$ ) and, per each parameter acquired, the percentage difference (accuracy) between the numerical output and the experimental value was obtained. As Table 6 shows, all the percentage values are less than $20 \%$.

\subsection{Tactile Flow for Softness Perception}

The concept of tactile flow, which mathematically describes the rate of expansion of isostrain volumes in human fingerpad, was introduced in [27] to provide a computational model for the explanation of some haptic perceptual illusions as well as for the mechanisms of human softness perception [27] However, despite many psychophysical evidences support the

Table 6. Force, Contact and Pressure Area Values comparison at three different levels of indentation $(1 \mathrm{~mm}, 2 \mathrm{~mm}$ and $3 \mathrm{~mm}$ ). Accuracy percentage value is reported as a comparison between the experimental and numerical output.

\begin{tabular}{|c|c|c|c|}
\hline \multicolumn{4}{|c|}{ Experimental and Numerical Values Comparison } \\
\hline Indentation & $1 \mathrm{~mm}$ & $2 \mathrm{~mm}$ & $3 \mathrm{~mm}$ \\
\hline $\begin{array}{c}\text { Experimental } \\
\text { Contact Area }\left[\mathrm{mm}^{2}\right]\end{array}$ & $87.3 \pm 15.31$ & $124 \pm 21.86$ & $133 \pm 13.10$ \\
\hline $\begin{array}{c}\text { Numerical } \\
\text { Contact Area }\left[\mathrm{mm}^{2}\right]\end{array}$ & 78.2 & 111 & 129 \\
\hline Accuracy [\%] & 10.4 & 10.4 & 3.00 \\
\hline $\begin{array}{c}\text { Experimental } \\
\text { Pressure Area }\left[\mathrm{mm}^{2}\right]\end{array}$ & $42.4 \pm 5.88$ & $75.4 \pm 13.1$ & $88.6 \pm 13.4$ \\
\hline $\begin{array}{c}\text { Numerical } \\
\text { Pressure Area }\left[\mathrm{mm}^{2}\right]\end{array}$ & 53.3 & 61.4 & 80.1 \\
\hline Accuracy [\%] & 20.4 & 18.5 & 9.6 \\
\hline $\begin{array}{c}\text { Experimental } \\
\text { Force }[\mathrm{N}]\end{array}$ & $0.78 \pm 0.03$ & $1.03 \pm 0.02$ & $1.71 \pm 0.06$ \\
\hline $\begin{array}{l}\text { Numerical } \\
\text { Force }[\mathrm{N}]\end{array}$ & 0.65 & 1.10 & 1.90 \\
\hline Accuracy [\%] & 16.6 & 6.63 & 10.0 \\
\hline
\end{tabular}

tactile flow model, a thorough numerical validation still lacks. The 3D FE model proposed in this work has been used to simulate the interaction between a fingerpad and a surface to verify if different levels of softness could be discriminated based on the rate of isostrain volume expansion in the .fingerpad. The results of our analysis reveals that it is possible to discriminate between two materials with different softness.

Both force-indentation and force-area plots are considered, as shown in Figure 10. As it is possible to notice, the curve for contact with the silicone material (red curve) shows lower forces, as one would expect, compared to the steel one (blue curve). Moreover, the force-area curve for silicone shows a higher CASR (Contact Area Spread Rate) with respect to the force-area curve for steel, which is coherent with [27].

\subsection{Model Estimation: Haptic Rendering}

The experimental data we collected have been then used to estimate the deformation model to reproduce the behaviour of finger skin for haptic rendering [23]. In particular, in order to reproduce the behaviour observed in the experimental measurements the experimental setup has been replicated together with the simulation of the response of a virtual finger being compressed by a flat surface. We aim for the simulated contact forces to be as similar as possible to the experimental ones. Moreover, we also replicate the boundary conditions of the experimental setup by positioning the finger in the same configuration and fixing the bottom nodes. During simulation, contact forces between the plane and the finger are computed using penalty forces with a stiffness value large enough to guarantee small penetrations. Figure 11 shows the finger model

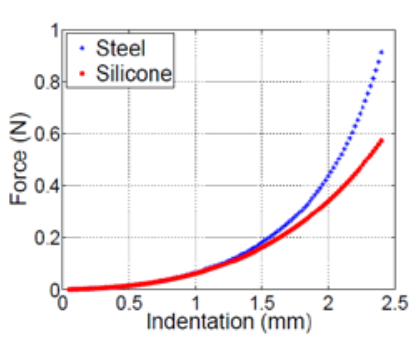

(a) Force-Indentation curve.

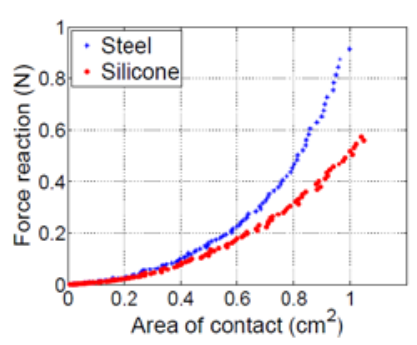

(b) Force-Area curve.
Figure 10. Force-area curves for the finger in contact with steel and silicone plate.

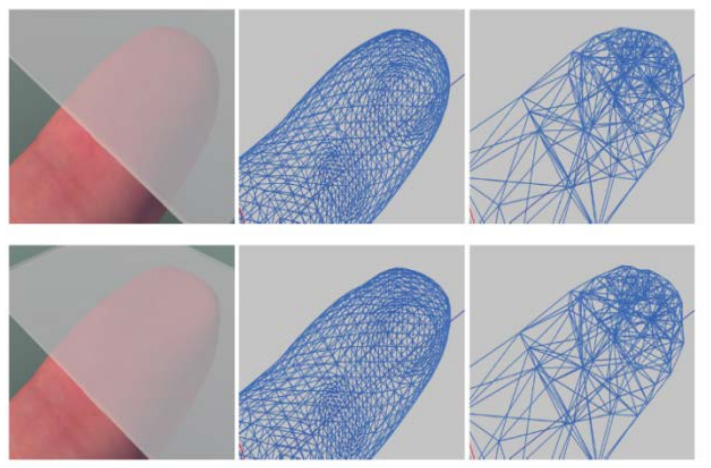

Figure 11. The images show two simulation steps of the finger in contact with the indenter surface during the estimation procedure. On top, in the undeformed configuration, and on the bottom, fingerpad deformed with a contact area of $150 \mathrm{~mm}^{2}$. Left: full render of the finger and the indenter; Middle: triangle mesh of the finger used for contact handling; Right: tetrahedral mesh of the finger used for deformation. 
[23] in its undeformed state and once deformed.

Figure 12 shows the contact area vs. contact force evaluation plot. The purely linear model in [23] averages the behaviour for small and large contact areas, but it appears too stiff for small contact areas and too soft as the contact area grows. We can also see how a nonlinear model separates from its linear component when constraints are activated around $100 \mathrm{~mm}^{2}$ contact area, and how the difference becomes larger as the contact area approaches $200 \mathrm{~mm}^{2}$.

Moreover, results demonstrate that an efficient and robust nonlinear model of skin mechanics can be estimated from controlled force and area experimental measurements. Also in this case, higher accuracy would require a thorough investigation material properties, such as viscosity and friction, as well as material heterogeneity.

\section{DISCUSSION AND CONCLUSIONS}

In this paper a preliminary integrated characterization of the human fingertip properties in terms of force $[\mathrm{N}]$, contact area $\left[\mathrm{mm}^{2}\right]$, contact pressure $\left[\mathrm{mm}^{2}\right]$, and indentation $[\mathrm{mm}]$, is presented. Using an innovative and non- invasive test rig device, we performed three experimental tests indenting the fingertip at three different levels of indentation $(1 \mathrm{~mm}, 2 \mathrm{~mm}$ and $3 \mathrm{~mm})$, while, only one single velocity value $(2 \mathrm{~mm} / \mathrm{s})$, as a preliminary test case, was considered.

The results, obtained from the experimental tests, are then compared with numerical outputs of a 3D Finite Model, which was built on previous 3D model in literature. Per each level of indentation ( $1 \mathrm{~mm}, 2 \mathrm{~mm}$ and $3 \mathrm{~mm}$ ) and, per each parameter acquired, the percentage difference (accuracy) between the numerical output and the experimental value was obtained.

Moreover, the here proposed FE model has been also used to numerically investigate the perceptual mechanism of human softness perception. Results, although preliminary, are encouraging and motivate us to proceed towards a more accurate, exhaustive and effective mechanical characterization of finger characteristics, with more subjects and more experimental test parameters, such as different levels of indentation and velocity. Finally, we show how our experimental data can be used for the estimation of the nonlinear deformation model for tactile rendering.

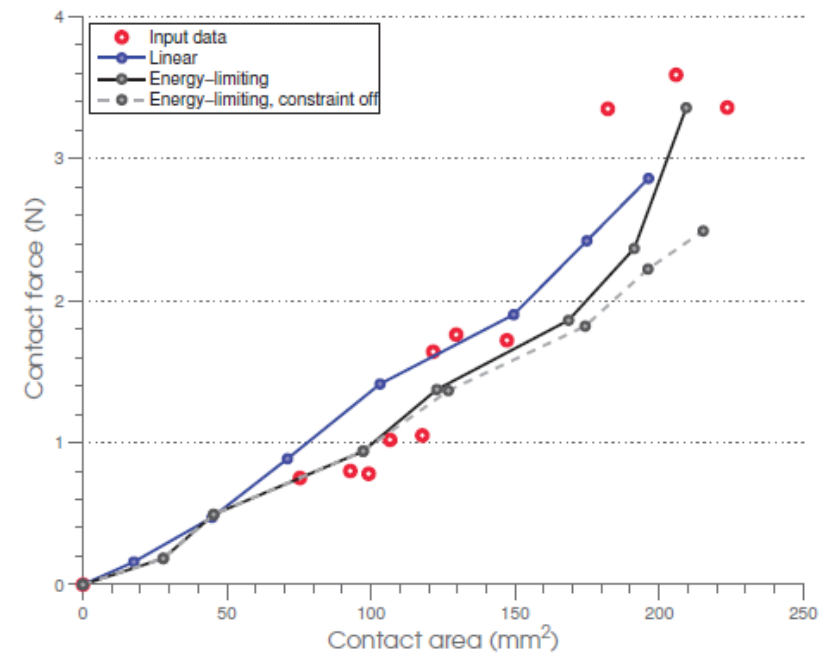

Figure 12. Evaluation of contact area vs. contact force for the estimated linear and energy-limiting models, as well as for the linear component.

\section{ACKNOWLEDGEMENT}

This work was supported in part by grants from the EU FP7 (project no. 601165 WEARHAP) and the European Research Council (ERC Advanced Grant no. 291166 SoftHands).

\section{REFERENCES}

[1] Johnson K. O., The roles and functions of cutaneous mechanoreceptors, (2001) Curr. Opin. Neurobiol., vol. 11, no. 4 pp. 455-461.

[2] Fruhstorfer H., Abel U., Garthe C. D., and Knuttel A., (2000) Thickness of the stratum corneum of the volar fingertips.

[3] Wu, J. Z., Dong, R. G., Rakheja, S., Schopper, A. W., Smutz, W. P. (2004). A structural fingertip model for simulating of the biomechanics of tactile sensation. Medical Engineering Physics, 26, 2, 165-175.

[4] Maeno, T., Kobayashi, K., Yamazaki, N. (1998). Relationship between the Structure of Human Finger Tissue and the Location of Tactile Receptors. Jsme International Journal Series C, 41, 1, 94-100.

[5] Levesque, V. (2004). Measurement of skin deformation using fingerprint feature tracking. Ottawa: National Library of Canada Bibliothèque nationale du Canada.

[6] Bowden, A. E., Rabbitt, R. D., Weiss, J. A., (1999). Stress and strain in the human distal phalanx under indentation. 1.

[7] Giavazzi, S., Ganatea, M. F., Suštarič, P., Rodič, T. (2010). Inverse determination of viscoelastic properties of human fingertip skin.

[8] Gulati, R. J., and M. A. Srinivasan. (1995). Human Fingerpad Under Indentation I: Static and Dynamic Force Response. ASME -PUBLICATIONS- BED. 29: 261.

[9] Srinivasan, M. A., LaMotte, R. H. (1991). Encoding of shape in the responses of cutaneous mechanoreceptors. Information Processing in the Somatosensory System: Proceedings of an International Symposium at the Wenner-Gren Center, 59-69.

[10] Peine, W. J., Howe R. D. (1998). Do humans sense finger deformation or distributed pressure to detect lumps in soft tissue?. in R.J.Furness, ed., Proc. of the ASME Dynamic System and Control Division, ASME International Mechanical Engineering Congress and Exposition, Anaheim, Nov. 19-20, 64, 273-278.

[11] Lee, J. W., Rim, K. (1991). Measurement of finger joint angles and maximum finger forces during cylinder grip activity. Journal of Biomedical Engineering, 13, 2, 152-162.

[12] Pawluk, D. T. V., Howe, R. D. (1999). Dynamic contact of the human fingerpad against a flat surface. Journal of Biomechanical Engineering, 121, 6, 605-611.

[13] Nakamura, T., Kimura, F., \& Yamamoto, A. (2013). A photoelastic tactile sensor to measure contact pressure distributions on object surfaces. Journal of Robotics and Mechatronics, 25, 2, 355-363.

[14] Dandekar, K., Raju, B. I., Srinivasan, M. A. (2003). 3-D finiteelement models of human and monkey fingertips to investigate the mechanics of tactile sense. Journal of Biomechanical Engineering, 125, 5, 682-691.

[15] Gerling, G. J., Rivest, I. I., Lesniak, D. R., Scanlon, J. R., Lingtian, W. J. R. (2014). Validating a Population Model of Tactile Mechanotransduction of Slowly Adapting Type I Afferents at Levels of Skin Mechanics, Single-Unit Response and Psychophysics. Ieee Transactions on Haptics, 7, 2, 216-228.

[16] Wu, J. Z., Krajnak, K., Welcome, D. E., Dong, R. G. (2008). Three-dimensional finite element simulations of the dynamic response of a fingertip to vibration. Journal of Biomechanical Engineering, 130(5) : 054501.

[17] Abdul H. A. R., Aladin Z., Begg R. K., \& Y. Wahab. (2012). Foot Plantar Pressure Measurement System: A Review. Molecular Diversity Preservation International.

[18] Yong, F., Yunjian, G., Quanjun, S., (2011) International Conference on Information and Automation (ICIA). A human 
identification method based on dynamic plantar pressure distribution. 329-332.

[19] http://www.sensorprod.com/automotive-pressure-mapping.php

[20] Shima, K., Tamura, Y., Tsuji, T., Kandori, A., Yokoe, M., Sakoda, S. (2009). Estimation of human finger tapping forces based on a fingerpad-stiffness model. Conference Proceedings : Annual International Conference of the Ieee Engineering in Medicine and Biology Society, 2663-2667.

[21] Bending stiffness of paper and paperboard, T 535 om-96 The Technological Association of the Pulp and Paper Industry.

[22] Pressurex - micro Green (http://www.sensorprod.com/pressurex micro.php)

[23] E. Miguel, M. L. D'Angelo, F. Cannella, M. Bianchi, M. Memeo, A. Bicchi, D.G. Caldwell and M. A. Otaduy. Characterization of nonlinear fingerpad mechanics for tactile rendering. World Haptics Conference (WHC), 2015 IEEE, Evanston, IL, 2015, pp. 63-68.
[24] Battaglia, E., Bianchi, M., D'Angelo, M. L., D'Imperio, M., Cannella, F., Scilingo, E. P., \& Bicchi, A. (2015). A Finite element model of tactile flow for softness perception. Conference Proceedings: Annual International Conference of the Ieee Engineering in Medicine and Biology Society, 2015, 24302433.

[25] Pawluk, D. T., Howe, R. D. (1999). Dynamic contact of the human fingerpad against a flat surface. Journal of Biomechanical Engineering, 121, 6, 605-11.

[26] ANSYS Academic Teaching Mechanical, Release 14.5.

[27] A. Bicchi, D. E. De Rossi, and E. P. Scilingo, (2000) "The role of the contact area spread rate in haptic discrimination of softness," IEEE trans. on Robotics and Automation, vol. 16, no. 5, pp. 496-504.

[28] M. Bianchi and A. Serio, "Design and characterization of a fabric-based softness display," IEEE Trans. Haptics, vol. 8, no. 2, pp. 152-163, Apr.-Jun. 2015. 